\section{Eyeing the AAV2 vector}

\section{By Michael J. Haas, Senior Writer}

A U.S. research team has shown that a gene therapy using a recombinant adeno-associated virus serotype 2 (AAV2) vector to treat Leber's congenital amaurosis did not induce serious immunological responses when delivered into the eyes of dogs and monkeys with previous exposure to AAV2. ${ }^{1}$ The findings bolster arguments that an ocular AAV2 vector-based gene therapy could safely treat humans with pre-existing immunity to the virus itself or the AAV2 vector.

Leber's congenital amaurosis (LCA) is a form of retinal degeneration caused by defects in 1 of at least 11 known genes. It leads to complete loss of vision by the time a patient reaches adulthood. Type 2 LCA results from defects in retinal pigment epithelium-specific protein 65kDa (RPE65).

The Children's Hospital of Philadelphia (CHOP) and Applied Genetic Technologies Corp. are conducting separate clinical trials of gene therapies-AAV2-hRPE65v2 and rAAV2CB-hRPE65, respectively-to treat type 2 LCA. Each therapy uses a different engineered AAV2 vector encoding human RPE65 delivered to the eye by subretinal injection.

CHOP's therapy is in a Phase I trial that excludes patients with high levels of serum anti-AAV2 antibodies; Applied Genetic's therapy is in a Phase I/II trial that does not have that exclusion criterion.

AAV vectors are generally considered safe because natural AAV infections in humans cause mild immune responses at most and are not known to cause any diseases. However, it has not been known whether AAV vector-based gene therapies could stimulate an immune response in individuals with prior AAV exposure and consequently adversely affect the therapy's efficacy or safety.

The U.S. team, led by Jean Bennett, scientific director of CHOP's LCA trial, set out to determine whether AAV2 vectors could indeed trigger immune responses that would hamper the effectiveness of AAV2-based gene therapy in dogs or monkeys with prior AAV2 exposure.

Bennett is also a professor of ophthalmology at the University of Pennsylvania and a member of CHOP's Center for Cellular and Molecular Therapeutics.

First, an AAV2 vector encoding RPE65 was delivered subretinally to one eye of AAV2-naïve dogs with type 2 LCA. The treatment improved vision in the treated eye, and it also elicited increased levels of anti-
AAV2 antibodies in the intraocular fluid of the treated eye and in blood serum compared with pretreatment baselines.

Treatment of the second eye two weeks later induced mild and transient inflammatory responses but still resulted in improved vision in the treated eye-indicating that pre-existing immunity caused by the first vector treatment had not compromised the effectiveness of the second vector treatment.

Next, the team repeated the subretinal gene therapy regimen in normal monkeys without ophthalmic disease that had been previously exposed to AAV2 vectors via intranasal, i.v. or intramuscular routes of delivery. Treatment elicited only mild immunological responses, which were comparable to those seen in the dogs.

Finally, the team determined that 41 of 100 healthy human subjects had detectable levels of serum anti-AAV2 antibodies, and patients with the highest levels of serum antibodies had only low or background levels in their intraocular fluid.

Collectively, the results suggest that subretinal administration of AAV2-based gene therapies in humans with previous exposure to the AAV2 vector or virus would not trigger intraocular immune responses and thus would still be effective.

The team included scientists from the University of Pennsylvania, the Howard Hughes Medical Institute, The University of Iowa and the University of Missouri-Columbia.

The results were published in Science Translational Medicine. ${ }^{1}$

\section{Safe outlook}

Ophthalmic researchers said the findings add to evidence that subretinal delivery of AAV vector-based gene therapies could safely treat humans who had preexisting AAV immunity-and in turn suggest that intravitreal delivery of those gene therapies might not cause serious immune responses, either.

"Our studies and studies by our collaborators in small animals told us that prior AAV2 exposure was not an issue," said Susan Washer, president and CEO of Applied Genetic. "The Bennett team's study nicely confirms that in larger animals."

Jeffrey Chulay, CMO and VP of regulatory affairs, noted that the trial of Applied Genetic's LCA gene therapy does not exclude patients with high levels of serum antibodies against the vector "because the eye is considered an immune-privileged site-meaning that immune responses outside the eye have little or no impact inside it."

But Washer also noted that the trial does exclude patients who have been previously treated with the vector. "This is somewhat of a holdover from earlier LCA trials-before there was good information on safetywhen specific treatment with a vector was thought to perhaps increase overall risk," she said.

Washer added: "In the future we may consider treating patients that have also been treated with a recombinant AAV gene therapy vector."

Jay Neitz, professor of ophthalmology at the University of Washing- 
ton, agreed that the Bennett team showed that it should be safe to readminister the vector to patients. "There will be times when you would want to give a subretinal injection multiple times to the same patient, whether to treat the second eye or treat the same eye with another gene therapy" that utilizes the vector, he said.

"Subretinal injection produces a small, temporary detachment of the retina and thus is slightly invasive, whereas intravitreal injection doesn't disturb the retina at all" and is less uncomfortable for the patient, he added.

Nevertheless, according to Neitz, subretinal injection has been the preferred route of delivering ophthalmic gene therapies because it is thought to incur less risk of an immune response than intravitreal injection. However, he said, "if injection into the subretinal space is safer than people have thought, then injection into the vitreal cavity might also pose less of a risk" than previously assumed.

Jay Neitz and Maureen Neitz, also a professor of ophthalmology at the University of Washington, head a research team that is evaluating whether subretinal injections of an AAV vector-based gene therapy could treat congenital achromatopsia, a condition in which patients see very poorly because of a complete lack of color vision. They are working in mouse models of the disease that they have developed.

\section{Line of sight}

Bennett said her team's ongoing work includes testing immunological responses in animals when the AAV2 vector-based RPE65 gene therapy is re-administered to the same eye.
She said her team's findings should extend to any subretinal gene therapy that uses an AAV2 vector. But, she cautioned, "there may be additional immune responses towards the transgene product, if the AAV2 vector were delivering a gene that encoded a secreted protein, not an intracellular protein like RPE65."

According to Bennett, a patent covering the gene delivery technology used in the paper has been issued to The University of Florida Research Foundation Inc. (UFRF), the Trustees of the University of Pennsylvania and Cornell University, and the technology is available for licensing from UFRF.

Haas, M.J. SciBX 3(11); doi:10.1038/scibx.2010.330

Published online March 18, 2010

\section{REFERENCES}

1. Amado, D. et al. Sci. Transl. Med.; published online March 3, 2010; doi:10.1126/scitranslmed.3000659

Contact: Jean Bennett, University of Pennsylvania, Philadelphia, Pa. e-mail: jebennet@mail.med.upenn.edu

\section{COMPANIES AND INSTITUTIONS MENTIONED}

Applied Genetic Technologies Corp., Alachua, Fla.

The Children's Hospital of Philadelphia, Philadelphia, $\mathrm{Pa}$.

Cornell University, Ithaca, N.Y.

Howard Hughes Medical Institute, Chevy Chase, Md.

The University of Florida Research Foundation Inc., Gainesville, Fla.

The University of lowa, lowa City, lowa

University of Missouri-Columbia, Columbia, Mo.

University of Pennsylvania, Philadelphia, $\mathrm{Pa}$.

University of Washington, Seattle, Wash. 